\title{
Repensar la educación online durante el confinamiento: la visión de las familias de Castilla y León
}

\section{Rethinking online education during lockdown: the view of families in Castile and León}

\author{
Enrique-Javier Díez-Gutiérrez ${ }^{1}$, Katherine Gajardo Espinoza ${ }^{2}$ \\ ${ }^{1}$ Universidad de León enrique.diez@unileon.es \\ ${ }^{2}$ Universidad de Valladolid katherine.gajardo@usach.cl
}

Recibido: $21 / 10 / 2020$

Aceptado: $20 / 4 / 2021$

Copyright (C)

Facultad de CC. de la Educación y Deporte.

Universidad de Vigo

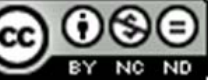

Dirección de contacto:

Enrique Javier Díez Gutiérrez

Facultad de Educación, Universidad de León,

Campus de Vegazana, s/n

24071 León

\begin{abstract}
Resumen
La crisis pandémica del COVID 19 obligó a cerrar todas las instituciones escolares y continuar la educación de forma online en España. Fue así como de un momento a otro, miles de estudiantes tuvieron que cambiar sus rutinas y modos de aprendizaje, hecho que impactó de formas diversas en las organizaciones familiares del país. En el presente estudio se expone la visión de las familias de la Comunidad Autónoma de Castilla y León sobre cómo afrontaron esta situación y su valoración respecto a las medidas tomadas por las administraciones educativas durante el estado de alarma y confinamiento obligatorio decretado desde marzo de 2020. La metodología del estudio es cuantitativa, con un planteamiento descriptivo. Se aplicó una encuesta online a una muestra representativa de 570 integrantes de unidades familiares de la Comunidad Autónoma y se realizaron análisis estadísticos descriptivos, contrastación de hipótesis y análisis de contenido cualitativo sobre la información recogida. Los resultados muestran la persistencia de brechas en el acceso a medios y recursos digitales en la población más vulnerable, la demanda generalizada por flexibilizar los procesos educativos y evaluativos que se están dando en las escuelas en una situación excepcional como la pandemia, y una visión crítica respecto a algunas políticas educativas adoptadas, que se han centrado en avanzar contenidos y examinarlos, algunas veces desatendiendo la diversidad de las y los estudiantes.
\end{abstract}

\section{Palabras clave}

Enseñanza Online, Evaluación del Estudiante, Familia, Educación Universal, COVID-19, Desigualdad Educativa

\begin{abstract}
The COVID 19 pandemic crisis forced the closure of all school institutions and promoted online learning in Spain. Thus, in a short time, thousands of students had to change their routines and ways of learning, a fact that impacted in different ways on family organizations in the country. This study presents the vision of families in the Autonomous Community of Castile and León on how they faced this situation and their assessment of the measures taken by the educational administrations during the
\end{abstract}


state of crisis and compulsory confinement decreed since March 2020. The methodology of the study is quantitative, with a descriptive approach. An online survey was applied to a representative sample of 570 members of family units in the Autonomous Community and descriptive statistical analysis, hypothesis testing, and qualitative content analysis were carried out on the information collected. The results show the persistent hurdles to access media and digital resources in the most vulnerable population, the generalized demand for making educational and evaluative processes more flexible in schools in exceptional cases such as the pandemic, and a critical view of some educational policies adopted, which have focused on advancing content and examining it, sometimes disregarding the diversity of students.

\section{Key Words}

Distance Learning, Universal Education, Family, Educational Assessment, COVID19, Educational Inequality

\section{INTRODUCCIÓN}

El 23 de marzo de 2020, la Consejería de Educación de la Comunidad Autónoma de Castilla y León anunciaba que los centros docentes se cerrarían de forma total, salvo en caso de "necesidades específicas", para garantizar la actividad docente no presencial, desde ese momento y mientras durara el estado de alarma decretado por el Gobierno de España, debido a la pandemia del Coronavirus.

Esto supuso que las comunidades educativas tuvieran que cambiar radicalmente de escenario para seguir desarrollando el proceso educativo. Más de 500.000 estudiantes de Castilla y León (MEFP, 2020) se vieron obligados a permanecer confinados en sus domicilios y continuar su proceso de formación vía online (Samaniego, 2020), pasando de una educación en instalaciones y espacios acondicionados expresamente para ello, con una metodología docente de interacción presencial, con recursos y medios, así como un seguimiento constante y un apoyo inmediato, a una situación no presencial y a distancia que era completamente nueva e inesperada (Cotino, 2020).

El estado de alarma se prolongó hasta el 7 de junio y con ello, todas las previsiones por parte de la Junta de Castilla y León eran que el curso académico 2019/2020 se daría por acabado sin actividad presencial y sin saber a ciencia cierta si el inicio del nuevo curso se podría hacer de forma presencial completa o se articularían formas híbridas de formación debido a la persistencia de la pandemia en septiembre de 2020.

El confinamiento en los hogares supuso tener que hacer frente a numerosas dificultades para las que muchos entornos familiares no estaban preparados (Heppen et al., 2017; UNESCO, 2020). No todas las familias tenían acceso a dispositivos tecnológicos o conexión a Internet para poder continuar con la educación online (Díez y Gajardo, 2020; UNICEF, 2020). A esto se le suma la disminución de la autorregulación, motivación y constancia de los estudiantes en la educación online. Las evidencias muestran que, incluso entre la población adulta, menos del 5\% de los inscritos completan los cursos online ofrecidos por Universidades prestigiosas (Bettinger, Fox, Loeb y Taylor, 2017; Xu y Jaggars, 2014).

A lo anterior se suman otras dificultades, como no tener un espacio adecuado para estudiar, la falta de formación de los progenitores para ayudar educativamente, el impacto de los roles de género asumidos en los núcleos familiares o las propias 
características socioeconómicas y culturales de las familias, entre otros, lo que amplifica todavía más la desigualdad educativa (Martín y Gómez, 2017; Cabrera, 2020) en una situación donde la escuela presencial deja de poder desempeñar un papel de compensación socioeducativa, sobre todo en aquellos entornos más necesitados (Martín y Rogero, 2020).

Esta situación obliga a repensar cómo afrontar la escolaridad cuando acecha una pandemia y cuando aún no sabemos si supondrá un cambio permanente en nuestras formas de convivencia (Piña-Ferrer, 2020). En este sentido es una necesidad conocer cómo viven y perciben las familias las medidas que los responsables políticos plantean tratando de equilibrar las condiciones de la salud pública con el mantenimiento de una educación online (García-García, 2020), para que el lema común adoptado de "que nadie quede atrás" sea una realidad.

Todo lo anterior nos lleva a preguntas urgentes como ¿en qué situación se encuentran las familias y estudiantes que están actualmente inmersas en este proceso de educación online?, ¿tienen los recursos necesarios?, ¿tienen los apoyos suficientes?, ¿cómo valoran las medidas tomadas para finalizar el curso?, ¿y de cara a la evaluación de este?, ¿qué opinan sobre las actividades en verano? y ¿cómo piensan que se debe hacer frente al inicio del próximo curso? ¿qué factores sociales inciden en su valoración?

Ante estas preguntas, las autoridades y responsables educativos se enfrentan a decisiones difíciles ante la situación de la población sometida a un largo estrés de confinamiento (AEPNA, 2020; Ceballos y Sevilla, 2020; Hernández, 2020; OzamizEtxebarría, Dosil-Santamaría, Picaza-Gorrochategui e Idoiaga-Mondragón, 2020). Por eso es importante ofrecerles evidencias de la investigación científica que les faciliten la toma de decisiones y que ayuden a afrontar estas situaciones de crisis con mayor probabilidad de éxito y de acierto.

En este caso, presentamos los resultados de un estudio sobre cómo las familias de Castilla y León perciben y valoran las medidas tomadas por las administraciones educativas de cara a los procesos de aprendizaje y el futuro de la educación en tiempos de pandemia. Para la realización de este estudio contamos con los datos cuantitativos y cualitativos recabados a través de una encuesta aplicada en España, con especial incidencia e impacto en Castilla y León.

\section{METODOLOGÍA}

\subsection{Objetivos}

Se ha pretendido conocer la valoración de las personas afectadas directamente por las medidas de confinamiento y supresión de la escolaridad presencial en tiempos de pandemia. En este contexto generamos un proyecto de investigación nacional, que desde el mes de marzo de 2020 ha estado realizando una investigación que identifique la situación educativa del país. Nuestra intención en estas líneas es exponer de forma descriptiva algunos de los hallazgos, describiendo la situación de los hogares respecto al proceso educativo y las valoraciones de las familias de Castilla y León sobre las medidas adoptadas para finalizar el curso escolar y comenzar el siguiente en esta situación excepcional. También conocer si existen diferencias significativas, en función 
del sexo y de la zona rural o urbana, en sus percepciones sobre el desarrollo adecuado del curso académico en tiempos de confinamiento.

\subsection{Instrumento y muestra}

La investigación se ha desarrollado mediante un diseño descriptivo, por medio de una encuesta electrónica. Aplicamos un cuestionario previamente validado por un método Delphi de consulta a personas expertas (Díez-Gutiérrez y Gajardo, 2020). El cuestionario está divido en cinco apartados: 1) seis preguntas de caracterización sociodemográfica; 2) cuatro preguntas sobre condiciones del hogar para poder seguir el proceso de educación online; 3) tres preguntas sobre el tipo de acompañamiento y apoyo académico que se ha llevado en el hogar durante el confinamiento; 4) seis escalas de conformidad sobre estrategias de educación y evaluación para finalizar el curso escolar; y 5) cuatro escalas de conformidad sobre acciones educativas de cara a iniciar el nuevo curso escolar. Para finalizar se presenta una pregunta abierta en la que los y las participantes pueden voluntariamente expresar libremente planteamientos y experiencias en medio del confinamiento.

Para la administración del cuestionario se utilizó un muestreo por cuotas (Mendieta, 2015; Hernández y Carpio, 2019). Se seleccionaron representantes de la población de Castilla y León por la variable "Sexo" y "Tipo de localidad de origen" basados en los datos del Instituto Nacional de Estadística ofrecidos en el portal de la Junta de Castilla y León (Estadística JCYL, 2020). Se eligió este método con la finalidad de conseguir una representación que pudiera aportar información relevante respecto a las características de la población familiar urbana y rural de Castilla y León.

La encuesta se aplicó de forma paralela a la investigación nacional y en dos fases, una entre el 18 y el 26 de abril de 2020 y otra desde el 28 de abril al 17 de mayo de 2020. El enlace al formulario de la encuesta se difundió por redes sociales (Facebook, Twitter e Instagram), publicaciones en sitios web de instituciones educativas de Castilla y León, mensajes públicos en grupos comunitarios (WhatsApp y Telegram) y mediante el envío de correos electrónicos masivos a todas las provincias de la Comunidad Autónoma (Serna, 2019).

Con el fin de verificar la validez externa de la muestra $(n=570)$, entendiendo la naturaleza no probabilística de ésta, se aplicaron los criterios recomendados por Reips (2002), primero por medio de la selección y descarte de los datos de determinados perfiles hasta obtener una muestra representativa, y segundo, observando las discrepancias entre dos grupos muestrales (de la fase 1 y la fase 2). Al realizar análisis no paramétricos de diferencias entre grupos no se encontraron diferencias significativas en las variables, con ello se aseguró la consistencia suficiente para garantizar la validez.

\subsection{Características de la muestra}

Una síntesis sobre las características de la muestra se presenta en la Tabla 1. 


\begin{tabular}{|c|c|c|}
\hline Sexo & Frecuencia & Porcentaje \\
\hline Mujer & 292 & 51,2 \\
\hline Hombre & 278 & 48,8 \\
\hline Tipo de localidad & Frecuencia & Porcentaje \\
\hline Zona rural de menos de 100 hab. & 11 & 1,9 \\
\hline Zona rural - población de entre 100 y 500 hab. & 63 & 11,1 \\
\hline Zona rural - población de entre 501 y 1000 hab. & 34 & 6,0 \\
\hline Zona rural- población de entre 1001 y 5000 hab. & 40 & 7,0 \\
\hline Zona rural - población de más de 5000 habitantes & 46 & 8,1 \\
\hline Zona urbana & 376 & 66,0 \\
\hline Ocupación & Frecuencia & Porcentaje \\
\hline Estudio & 61 & 10,7 \\
\hline Trabajo & 428 & 75,1 \\
\hline Desempleo & 48 & 8,4 \\
\hline Pensionista & 24 & 4,2 \\
\hline Ama o amo de casa & 5 & 0,9 \\
\hline Excedencia & 4 & 0,7 \\
\hline Edades & Frecuencia & Porcentaje \\
\hline $17-20$ años & 25 & 4,5 \\
\hline 21-24 años & 19 & 3,3 \\
\hline 25-29 años & 23 & 4,0 \\
\hline 30-34 años & 29 & 5,1 \\
\hline 35-39 años & 59 & 10,4 \\
\hline 40-44 años & 107 & 18,8 \\
\hline 45-49 años & 120 & 21,1 \\
\hline $50-54$ años & 100 & 17,5 \\
\hline 55-59 años & 51 & 8,9 \\
\hline Más de 60 años & 37 & 6,5 \\
\hline Estudios & Frecuencia & Porcentaje \\
\hline Estudios primarios & 14 & 2,4 \\
\hline Secundaria Obligatoria & 56 & 9,9 \\
\hline Bachillerato & 106 & 19,1 \\
\hline Formación Profesional & 270 & 47,0 \\
\hline Diplomatura & 41 & 6,7 \\
\hline Licenciatura o Grado & 53 & 8,5 \\
\hline Postgrado & 33 & 6,4 \\
\hline
\end{tabular}

Tabla 1. Caracterización de la muestra

La representación de hombres (49\%) y mujeres (51\%) es proporcionalmente igual a la de la población de Castilla y León, al igual que las características de la localidad de origen: $66 \%$ de los y las encuestadas provienen de entornos urbanos de más de 5.001 habitantes, mientras que el $34 \%$ proviene de entornos rurales de distinta cantidad poblacional. 
Los y las representantes de las unidades familiares suelen ser principalmente personas trabajadoras (75\%), con edades que varían entre los 35 y 54 años. Respecto a $\mathrm{su}$ formación existe presencia mayoritaria de quienes han alcanzado la titulación correspondiente a los estudios obligatorios y han decidido seguir una Formación Profesional. Llama la atención la presencia de un $11 \%$ de encuestados y encuestadas que no trabajan activamente. De este porcentaje nos encontramos con un $8 \%$ que declara estar en condición de desempleo.

\subsection{Análisis de datos}

Los análisis fueron realizados con apoyo del paquete IBM® SPSS Statistics versión 26 y se centraron en estadísticos descriptivos, frecuencias y contrastes de hipótesis. La base de datos inicial fue exportada y sus variables y casos codificados. La muestra inicial se compuso por 1.597 casos los cuales, luego de la identificación y eliminación de casos duplicados y casos con valores perdidos en más de cuatro variables y la aplicación de una selección aleatoria de perfiles para obtener cuotas representativas (Reips, 2002), se tradujeron en una muestra final de 570 casos.

Para analizar la pregunta abierta del cuestionario se utilizó el método de análisis de contenido cualitativo de Abela (2002). Cada uno de los 178 textos escritos por los y las participantes fueron exportados a documentos independientes, los cuales se integraron a una unidad hermenéutica del programa informático Atlas TI. versión 8. Se realizó una codificación deductiva e inductiva sobre la base del modelo generado por Díez y Gajardo (2020), y, con soporte del programa, los códigos se sometieron a saturación temática. A partir de esta última acción se identificaron dos dimensiones divididas en seis categorías temáticas.

\section{ANÁLISIS Y RESULTADOS}

\subsection{Descripción de las condiciones del hogar durante el confinamiento}

Las personas encuestadas ofrecieron información sobre las condiciones de sus hogares en el momento del inicio y desarrollo del confinamiento. La información brindada se puede sintetizar así:

- Un $6 \%$ de la muestra corresponde a personas solas; principalmente hombres trabajadores de sectores urbanos con edades comprendidas entre los 35 y 59 años.

- Un $57 \%$ de la muestra vive con una persona más; este perfil corresponde principalmente a hombres trabajadores de sectores urbanos con edades comprendidas entre los 40 y los 54 años.

- Un 38\% de la muestra vive con dos o más personas durante el confinamiento; principalmente a mujeres trabajadoras de entornos rurales con edades entre los $50 \mathrm{y}$ 59 años.

- Un 67\% de la muestra ha pasado su confinamiento teniendo a cargo al menos un niño o niña. De este porcentaje el $56 \%$ de los casos dice tener solo un niño o niña a cargo $(n=144)$. Los perfiles con mayor representación respecto a la convivencia con menores son: 1) trabajadoras con edades comprendidas entre los 40 y los 50 años, habitantes de zonas urbanas; 2) trabajadores de entre 40 y 54 años, habitantes 
de zonas urbanas; y 5) trabajadoras de zonas rurales de entre 100 y 500 habitantes, con edades entre 35 y 39 años.

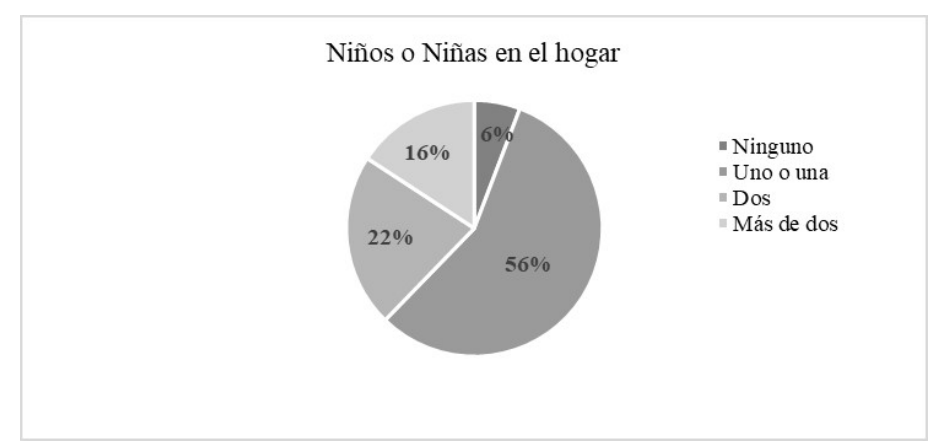

Figura 1. Distribución de respuesta a la pregunta ¿Con cuántos niños o niñas ha compartido el confinamiento en su hogar?

En cuanto al acceso a material básico para la educación, quienes participan en el estudio señalan en su gran mayoría que cuentan con los medios necesarios para una educación online:

- Respecto al acceso a teléfonos móviles, un 90\% de los encuestados declaran tener acceso a dos o más en el hogar. Un 9,8\% dispone de al menos un teléfono y solo un caso expone no tener acceso a uno.

- Si hablamos del acceso a ordenadores o portátiles en el hogar, un $27 \%$ de los encuestados declara tener más de dos, un 38\% declara tener dos, un 31\% declara tener 1 y un $4 \%(n=22)$ no posee ordenador o portátil en el hogar.

- Relativo al acceso a impresora en el hogar, un 67\% señala tener acceso a 1 ó 2, el $33 \%$ restante no tiene acceso a impresora.

- Respecto al acceso a Internet en el hogar, un 68\% de los encuestados posee una conexión rápida y/o estable, un $29 \%$ posee una conexión lenta y/o inestable y el $2 \%$ restante no tiene acceso a internet en el hogar.

En cuanto a los espacios físicos del hogar adaptados para el trabajo vemos que la mitad de los y las participantes tienen siempre un espacio disponible y la otra mitad cuenta con él a veces o directamente no lo tiene:

- Un 51\% posee acceso siempre a un espacio independiente y silencioso para poder trabajar, un $41 \%$ posee acceso a dicho espacio a veces y un $8 \%$ no posee un sitio adecuado para estudiar en el hogar.

En cuanto al acceso a materiales y medios suficientes para realizar el trabajo escolar y/o el estudio en el hogar:

- El $60 \%$ declara tener acceso a medios suficientes, un 34\% señala que a veces y dependiendo del material tienen acceso o no, y un $6 \%$, o sea 35 personas, no posee medios y materiales en su hogar para trabajar y/o estudiar.

\subsection{Seguimiento del curso académico desde el hogar}

Se identifica (Figura 2) un gran porcentaje de encuestados y encuestadas que advierten que las personas adultas del hogar no poseen tiempo o es insuficiente para acompañar en la realización de tareas a los más pequeños: 
Disposición de tiempo para el acompañamiento educativo

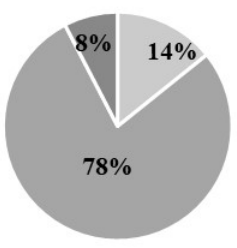

No $=$ A veces $=$ Sí

Figura 2. Respuesta a la pregunta ¿Las personas adultas han dispuesto de tiempo para acompañar a los más pequeños en su proceso educativo y en la realización de sus tareas?

A diferencia del anterior indicador, se observa un alto porcentaje (Figura 3) de quienes consideran que su formación y preparación es suficiente para enfrentarse al acompañamiento y apoyo en las tareas escolares que se les proponen a los menores desde la escuela.

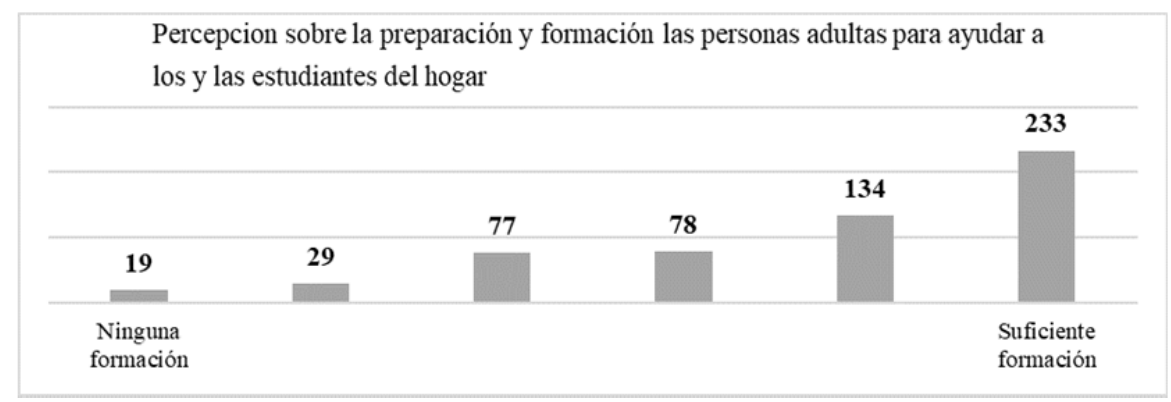

Figura 3. Respuesta a la pregunta ¿Crees que tienen suficiente preparación y formación las personas adultas de tu hogar para ayudar a los que están en edad escolar a realizar las tareas escolares?

La percepción respecto a si en el entorno familiar se ha podido seguir de forma adecuada el proceso educativo online durante el confinamiento generó una escala de percepciones diversa (Figura 4). Las personas encuestadas tienen opiniones divididas respecto a si las actividades educativas online se han podido desarrollar de la forma esperada y si se les ha dado una respuesta adecuada.

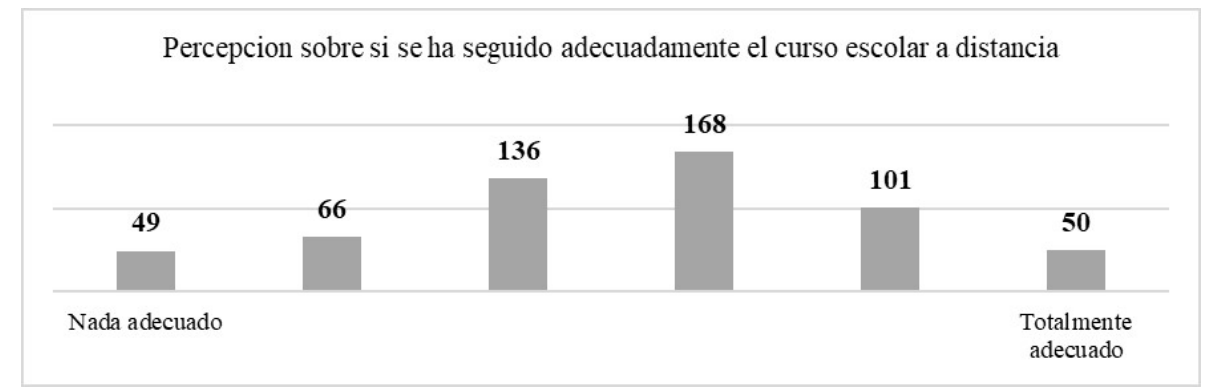

Figura 4. Respuesta a la pregunta ¿Crees que con las circunstancias vividas en este tiempo se ha podido seguir el curso escolar a distancia de forma adecuada? 


\subsection{Valoraciones sobre una futura educación y evaluación en tiempos de pandemia}

Cuando se les preguntó a los y las participantes del estudio si consideraban necesario y adecuado avanzar más contenidos del currículum escolar durante el tercer trimestre, en el caso de no volver a la escuela hasta el próximo curso, las respuestas obtenidas se resumen así:

- Un 59\% apuesta porque el trimestre se destine fundamentalmente a recuperar y reforzar los contenidos ya desarrollados en los trimestres anteriores. Mientras que un $34 \%$ considera que se debe avanzar con el currículum, continuando con los contenidos propios del tercer trimestre que no se habían abordado en los dos trimestres anteriores. Un grupo sustancialmente menor $(2,8 \%)$ aboga por combinar ambas opciones (repasar lo esencial y seguir avanzando en nuevos contenidos simultáneamente) y el grupo restante señala no saber qué opción sería la mejor.

- Es significativo que un $66 \%$ de quienes han sido encuestados no está de acuerdo con que se pongan exámenes para evaluar durante el tercer trimestre en situación de confinamiento. Por el contrario, un $28 \%$ sí está a favor de que se realicen exámenes online sobre los contenidos del tercer trimestre y se califique en función de los resultados obtenidos en ellos. Las personas restantes declararon no saber qué opción es mejor.

- Resaltar que un alto porcentaje de personas, específicamente un 58\%, plantea que solo se debería valorar en positivo lo que se haya realizado durante el trimestre de confinamiento, de tal forma que no se penalice al alumnado.

- Además, un $32 \%$ propone que se evalúe lo realizado durante este trimestre, pero haciendo una media con los trimestres anteriores. Por último, un $9 \%$ se decanta por no tener en cuenta la calificación del tercer trimestre y solo valorar los dos trimestres anteriores.

Finalmente, al preguntarles su grado de acuerdo con las medidas para tomar de cara al inicio del curso siguiente, y sobre qué aspectos habrá tener en cuenta en la evaluación de cara a afrontarlo con garantías, observamos diferentes niveles de acuerdo respecto a las afirmaciones que hacían referencia a esos aspectos (Figura 5).

Destacó un alto grado de acuerdo en la necesidad de dotar de más docentes y reducir las ratios por grupo en cada clase para afrontar el nuevo curso desde una perspectiva no solo de seguridad sanitaria, sino desde un enfoque inclusivo, de atención a la diversidad y de una educación personalizada. También un significativo acuerdo respecto a dedicar los primeros días del curso que viene a abordar de forma pedagógica la situación pandémica vivida.

Los aspectos en los que ha habido más dispersión y menos acuerdo en cuanto a las respuestas han sido los referidos a la evaluación y promoción escolar. La propuesta de las administraciones educativas de que la promoción de curso fuera la norma general y la repetición una práctica excepcional no ha sido mayoritariamente apoyada por las personas encuestadas. 


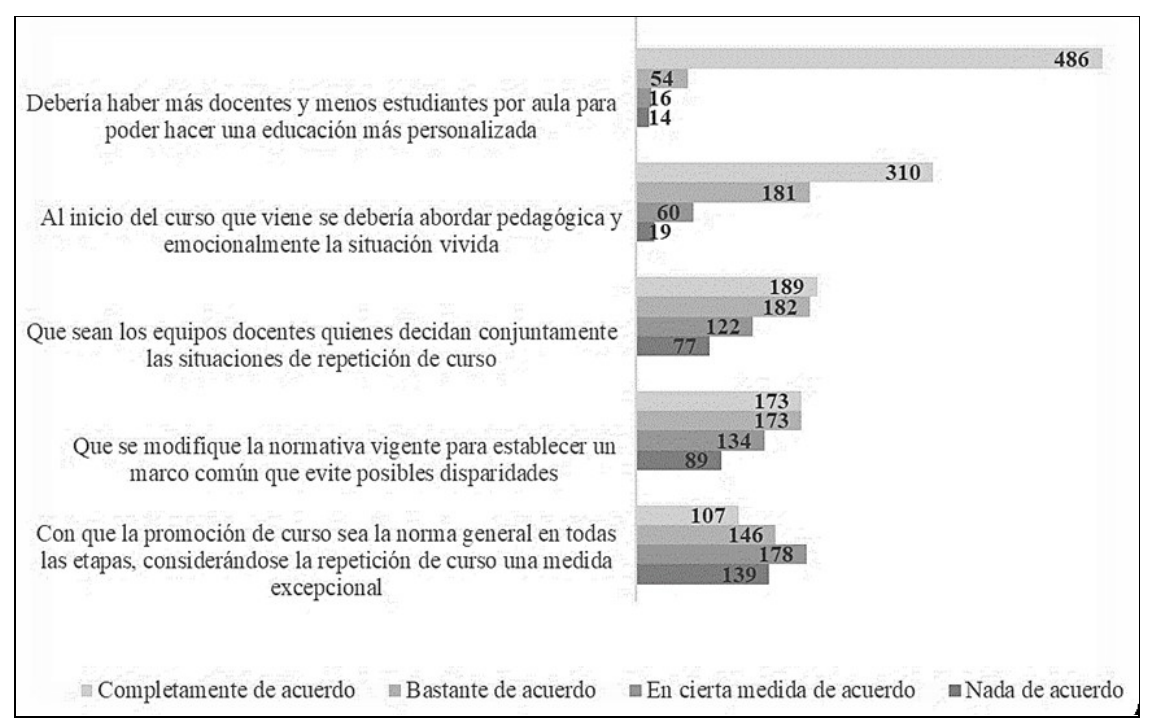

Figura 5. Respuesta a la pregunta ¿En qué medida estás de acuerdo con las siguientes acepciones?

\subsection{Relaciones entre las variables}

En este tercer bloque se pretende identificar si existen relaciones entre las variables, atendiendo a la distribución no normal de la muestra. Para ello realizamos la prueba $\mathrm{U}$ de Mann-Whitney (error al 0,05) para comparar la escala que medía las percepciones sobre el seguimiento del curso escolar con las variables de agrupación sexo (MujerHombre) y tipo de localidad de origen (Urbana y Rural).

\begin{tabular}{lllll}
\hline \multirow{2}{*}{ Escala } & Sexo & \multicolumn{2}{c}{ Localidad de Origen } \\
\cline { 2 - 5 } & U de MannWhitney & Sig. & U de MannWhitney & Sig. \\
\hline $\begin{array}{l}\text { Se ha podido seguir el curso } \\
\text { escolar a distancia de forma } \\
\text { adecuada }\end{array}$ & 37338,000 & 0,090 & 35174,500 & 0,475 \\
\hline
\end{tabular}

Tabla 2. Estadístico de contraste en función variables sexo y tipo de localidad de origen

De acuerdo con los datos (Tabla 2), no se identificaron diferencias significativas en la relación entre las variables, por lo cual no podemos afirmar que el sexo o el tipo de localidad de origen incidan en las percepciones sobre el desarrollo adecuado del curso académico en tiempos de confinamiento.

3.5. Temas más abordados respecto a educación en tiempos de confinamiento

En este cuarto apartado de resultados, ofrecemos una revisión de los temas emergentes que más aparecen en las respuestas a la pregunta abierta.

178 participantes contestaron, los textos tuvieron una media de 62 palabras y abarcan dos grandes dimensiones que se separan en categorías (Tabla 3). La dimensión 1 expone todas aquellas expresiones que hacen referencia a la educación y la evaluación durante el confinamiento; la dimensión 2 representa todas aquellas expresiones que hablan sobre la educación y la evaluación de cara al futuro inicio del nuevo curso escolar. 


\begin{tabular}{cl}
\hline \multicolumn{1}{c}{ Dimensiones } & \multicolumn{1}{c}{ Categorías } \\
\hline $\begin{array}{c}\text { 1. Educación y evaluación } \\
\text { ahora }\end{array}$ & $\begin{array}{l}\text { 1. Experiencias educativas personales o familiares durante el confinamiento } \\
\text { 2. Críticas a la labor docente y la escuela } \\
\text { 3. Propuestas para la evaluación de las actividades desarrolladas durante el } \\
\text { confinamiento. } \\
\text { 4. Críticas a las decisiones tomadas para afrontar la crisis }\end{array}$ \\
\hline $\begin{array}{c}\text { 2. Educación y evaluación } \\
\text { en el futuro }\end{array}$ & $\begin{array}{l}\text { 1. Propuestas educativas para el próximo curso. } \\
\text { 2. Propuestas de evaluación educativa para el próximo curso. }\end{array}$ \\
\hline
\end{tabular}

Tabla 3. Dimensiones y categorías identificadas

\subsubsection{Educación y evaluación en tiempos de pandemia ahora}

Respecto a la experiencias educativas personales o familiares durante el confinamiento en 25 casos los participantes eligen desarrollar textos narrativos que exponen experiencias y vivencias personales:

“Como profesorado nos estamos encontrando con un gran obstáculo: la mala calidad de las conexiones a internet y sin embargo estamos trabajando más que en el curso normal si eso cabe, para llegar a nuestros alumnos y responder a sus necesidades” (Mujer, Localidad Rural).

"Nuestro colegio solo se dedica a mandar una ficha al día, sin contacto con profesores y compañeros. Para los niños en casa sería de gran ayuda emocional poder compartir algún ratito con sus compañeros y profesores" (Mujer, Localidad Urbana).

"En mi caso personal, mis profesores no se han molestado en saber si sus alumnos/as tienen dispositivos, conexiones, y lo más importante si tienen conocimientos de informática y ofimática. Aunque ahora, después de un mes han mandado una encuesta para saberlo" (Hombre, Localidad urbana).

“Todo ha sido problemas ahora, cada profesor ha utilizado una aplicación distinta para dar clase. El aula virtual de la JCyL siempre está saturada por la mañana (...) todo un caos para alumnos y padres que hemos tenido que descargar en mi caso hasta 6 aplicaciones” (Hombre, Localidad Urbana).

Como se observa en estos ejemplos, la mayoría de los textos narrativos relatan experiencias negativas relacionadas con el acceso a los recursos educativos y su uso, al igual que reflexiones sobre la incapacidad propia o ajena para ofrecer alternativas adecuadas.

Por otro lado, se observan variadas críticas a la labor docente y la escuela, en 21 casos se vierten críticas respecto a la labor del profesorado y las instituciones educativas para afrontar la crisis con medidas educativas que no sean un mero traslado de las tareas tradicionales escolares al formato online, cuestionado la acumulación y saturación de "deberes" que envía el profesorado y remarcando que se pierde o no se tiene en cuenta la atención a la diversidad y al alumnado con más dificultades:

“Bajo mi humilde opinión les falta vocación (al profesorado) y motivación, no se vuelcan con los niños más rezagados o con dificultades para el aprendizaje, no se puede cargar al alumnado con deberes para casa porque no lo hayan hecho en clase. Los padres somos eso, padres, no profesores” (Mujer, Localidad rural).

"Hay profesores de bachiller machacando a los alumnos días no lectivos con llamadas y exámenes generando más estrés y ansiedad que el que ya estamos viviendo las familias confinadas" (Hombre, Localidad urbana). 
"Los profesores han seguido cobrando su salario, no han entrado en el sufrimiento de un ERTE (...) una buena mayoría NO ha aprendido a utilizar las herramientas gratuitas puestas a su disposición y pagadas con nuestros impuestos” (Mujer, Localidad urbana).

"Los equipos directivos no han logrado asumir su rol de brújulas en esta etapa... y también se puede decir que eso se debe a la falta flagrante de unas normas que seguir" (Mujer, Localidad rural).

Como se observa en los ejemplos, el perfil predominante que expone este tipo de apreciaciones corresponde a padres y madres que desarrollan argumentos negativos respecto al rol que se ven obligados a asumir durante el confinamiento y la situación de estrés que les genera también a ellos esa saturación de tareas demandadas por el profesorado. A diferencia del apartado anterior no narran situaciones, sino que expresan directamente sus valoraciones.

También los participantes generan propuestas sobre cómo realizar la evaluación de las actividades desarrolladas durante el confinamiento, en concreto 12 personas hacen reflexiones y propuestas en este sentido:

"Para evaluar hay que tener en cuenta la situación de las familias, hay familias sin medios, pero también las hay con todos los medios y no colaboran. Su profesorado conoce cada situación, por ello debería tenerse en cuenta su opinión” (Mujer, Localidad urbana).

"Mi opinión, es que se debería hacer media de los dos trimestres primeros y valorar el tercer trimestre con las tareas y deberes realizados avanzando materia” (Hombre, Localidad rural).

"Evaluar ahora es una situación especialmente dura para las familias con niños con dificultades, creo que va a ser necesario que el profesorado se ciña totalmente a los expedientes” (Mujer, Localidad rural).

Mayoritariamente las propuestas expresadas de forma libre, como se expone en estos ejemplos, tienden a plantear medidas de evaluación y promoción en sentido positivo que flexibilicen la normativa y no penalicen al alumnado por esta situación tan anómala que se está viviendo y considerando que se tome en cuenta la heterogeneidad del alumnado y se flexibilicen los sistemas de evaluación mientras dura el confinamiento.

Finalmente se evidenciaron algunas críticas a las decisiones políticas tomadas para afrontar la crisis, particularmente en 17 textos:

"A nuestros políticos lo único que les importa es que los niños y adolescentes de este país pierdan contenidos. Es francamente vergonzoso y deprimente en manos de quién estamos" (Mujer, Localidad urbana).

"Hay muchas cosas que debería haber evitado el Ministerio. Pero como todos sabemos el desconocimiento no exime de culpa. La falta de ilusión y de compromiso se ha hecho patente, y ahora, dejando en las mismas manos la responsabilidad de avanzar en la educación (...) destruyendo carreras e ilusiones de nuestros hijos, creo que volveremos a cometer un grave error" (Mujer, Localidad urbana).

"Creo que son momentos difíciles y que, en este caso, lo importante son los alumnos, no lo bien que quedan los políticos, así que ellos deberían contar más con nuestra opinión directa” (Hombre, Localidad urbana).

La mayoría de los textos valoran de forma negativa las decisiones de políticos y responsables educativos, reclamando que muchas veces se toman sin contar con ellos. 


\subsubsection{Educación y evaluación en tiempos de pandemia en un futuro incierto}

En esta dimensión temática señalan propuestas sobre cómo se debería afrontar el inicio del próximo curso.

Respecto a las propuestas educativas para el próximo curso en 25 textos las personas recomiendan acciones que desde su punto de vista podrían ser efectivas:

“En las zonas rurales no tenemos las opciones que en la ciudad (...) se debiera apostar por la apertura de los colegios rurales en verano, este año especialmente, pero todos los años en general, en el futuro se puede poner un servicio de conciliación a las familias rurales, así como un refuerzo educativo para los niños” (Mujer, Localidad Rural).

"Debiera haber una reordenación clara de todo el currículo de cara al año escolar siguiente” (Hombre, Localidad urbana).

"Hará falta que siempre haya menos alumnos por profesor y que se sigan usando las nuevas tecnologías, que se prepare para el futuro” (Mujer, Localidad urbana).

Gran parte de las propuestas sobre cómo iniciar el próximo curso plantean actividades de refuerzo y de apoyo para todo el alumnado, tras el confinamiento, pero especialmente dirigidas a quienes tuvieron más dificultades, así como utilizar los espacios e instalaciones escolares durante el verano o implementar servicios de conciliación laboral y familiar que ayuden en este proceso. También señalan la necesidad de aprender a discriminar lo esencial de lo prescindible en el currículo escolar, centrándose en aquello que es importante más que en la acumulación de contenidos. Reforzando el próximo curso con más profesorado, que como señalan algunas de las respuestas libres es "lo esencial en la escuela".

Por otra parte, en 10 de los textos las personas señalan propuestas de evaluación educativa para el próximo curso:

“Considero que se debería tener en cuenta en los criterios de evaluación que el alumnado no está preparado para esta situación y que el estrés generado se va a ver reflejado en el rendimiento académico" (Mujer, Localidad urbana).

"Creo que deberían quitar temas de la EBAU y estar en igualdad las diferentes CCAA” (Hombre, Localidad rural).

"Propondría seguir la evaluación online el próximo curso escolar hasta que haya vacuna. Los profesores que más lo necesiten y el alumnado podrían ponerse al día en TICs durante el Verano" (Mujer, Localidad urbana).

La mayoría de las propuestas avanzan hacia un modelo híbrido que concilie la evaluación online y la presencial. No obstante, también se señalan en algunas ocasiones los impactos negativos que pueden acarrear las evaluaciones negativas en aquellas personas que salen de una experiencia de confinamiento.

\section{DISCUSIÓN Y CONCLUSIONES}

Hemos comprobado que las condiciones de los hogares en Castilla y León son similares a las de los hogares de España (Díez y Gajardo, 2020; Fundación Telefónica, 2020), donde se han visto recluidos 10 millones de estudiantes $(8,5$ de enseñanzas no universitarias). 
Vemos que sigue existiendo población sin acceso a tecnología, tal como se reflejaba en la última encuesta del Instituto Nacional de Estadística sobre el uso de la tecnología, en 2019 , donde se indicaba que el $11 \%$ de los menores de 15 años no tienen acceso a un ordenador (INE, 2019) y el 8\% de los menores de 10 a 15 años no disponen de acceso a internet desde sus hogares tal como documentaba Unicef (EU Kids On Line, 2018). Según la Fundación Telefónica (2020) en Castilla y León, a fecha de 2019, el 89\% de hogares dispone de acceso a internet, lo cual contrasta con otras Comunidades Autónomas, como Galicia, donde se ha detectado que dos de cada tres alumnos y alumnas tienen que compartir ordenador con sus padres y hermanos, y cerca del 33\% no tiene acceso a Internet de calidad (CONFAPA, 2020). Esta brecha digital tiene que ver con la brecha económica y social, pues, según el informe PISA 2018, si en el nivel socioeconómico bajo, el $14 \%$ del alumnado no tiene ordenador en casa y un $44 \%$ solo tiene un ordenador que compartir con otros miembros del hogar, en el grupo socioeconómico alto, el $61 \%$ dispone de tres o más ordenadores en casa (Zubillaga y Gortázar, 2020, p. 8).

Las brechas (digital, económica y social) se ven incrementadas por las brechas culturales y de tiempo disponible respecto al apoyo y acompañamiento que los y las menores pueden recibir por parte de sus familias. Se observa que aquellas familias con cierto nivel cultural se sienten capaces de educar en el hogar pero que no poseen tiempo para hacerlo de forma eficiente. Se han detectado incluso casos en los cuales no hay acceso, material o espacios adecuados para trabajar y aprender en casa. Si bien estadísticamente son inferiores, existen y no deben ser invisibilizados. No nos olvidemos que, ya antes de la crisis sanitaria y social, uno de cada cuatro niños y niñas españoles estaba en riesgo de pobreza, y era la educación su único posible ascensor social (Lacort, 2020).

En cuanto a las medidas tomadas y recomendadas por las familias en este estudio, es relevante que se tienda a considerar necesario flexibilizar tanto el proceso de aprendizaje como la forma de evaluación y se insista en centrar los esfuerzos en desarrollar el proceso educativo desde un planteamiento inclusivo, con el fin de no dejar a nadie atrás, implementando medidas de apoyo y refuerzo desde el minuto cero, especialmente para el alumnado que más lo necesite, sin descuidar también el cuidado y el bienestar emocional de los menores ante el impacto de la pandemia.

Debemos analizar las consecuencias de un proceso de implementación tan acelerado de la educación online, como el que se ha desarrollado a consecuencia de la pandemia, y discutir la necesidad de repensar si hubiera sido necesaria y sigue siendo aún necesaria una planificación calmada de cómo afrontar pedagógicamente la educación online. Es incuestionable el valor de la educación online, como una herramienta complementaria a la educación presencial. Pero está claro que un modelo de educación online no puede sustituir a uno presencial, especialmente en las etapas de Infantil, Primaria y Secundaria obligatoria. El contacto, la relación directa, la comunicación, la interacción, la convivencia y la emoción son claves y esenciales en el proceso de enseñanza y aprendizaje de este periodo de la vida y esto solo se puede realizar presencialmente (Díez-Gutiérrez, 2021). Esta es una de las cuestiones que debemos resolver con serenidad y desde un planteamiento que garantice el pleno derecho a la educación.

Señalar también que existen críticas a la labor docente, centradas especialmente en el exceso de contenidos o tareas durante este tiempo tan sensible; críticas a la escuela, en cuanto a la disparidad mostrada en la atención escolar por parte de sus componentes - 
sobre todo la atención a la diversidad- y a las decisiones tomadas por la administración educativa para afrontar la crisis — relacionadas con la falta o la tardía reacción para compensar la escasez de recursos de las familias, los fallos de las plataformas educativas de trabajo online o la inacción-. En contraposición, se sigue valorando que sea el equipo de profesorado quien tome las decisiones respecto a la evaluación y la promoción de los y las estudiantes.

Finalmente, hay que indicar que algunas de las propuestas de cara a cómo afrontar la educación y la evaluación post-pandemia no se orientan en el mismo sentido que las actuales decisiones de la Comunidad Autónoma, como hemos visto en las opiniones que vertían los participantes de este estudio, lo cual nos lleva a preguntarnos si a la hora de tomar decisiones sobre las políticas educativas que les afectan ¿se está escuchando suficientemente a las familias y al alumnado que son una parte fundamental y esencial de la comunidad educativa?

En cuanto a las lecciones aprendidas que se extraen de los resultados, y de cara a la mejora de los centros educativos, hay que señalar la necesidad de que las administraciones educativas doten de suficientes recursos tecnológicos a los centros educativos, pero también de una formación continuada del profesorado y la comunidad educativa para utilizarlos pedagógicamente y compartir las buenas experiencias educativas en este sentido y los materiales digitales elaborados por la comunidad docente, potenciando especialmente el software libre y los recursos abiertos, como forma de avanzar hacia la soberanía digital y reducir la dependencia de las plataformas tecnológicas de las grandes multinacionales de la tecnología.

\section{BIBLIOGRAFÍA}

Abela, J.A. (2002). Las técnicas de análisis de contenido: Una revisión actualizada. Sevilla: Fundación Centro de Estudios Andaluces.

AEPNA. (2020). COVID-19, crisis y respuesta en salud mental. Revista de Psiquiatría InfantoJuvenil, 37(1), 34. https://doi.org/10.31766/revpsij.v37n1a1

Bettinger, E.P., Fox, L., Loeb, S. y Taylor, E.S. (2017). Virtual classrooms: How online college courses affect student success. American Economic Review, 107(9), 28, 55-75. http://doi.org/gbzjmd

Cabrera, L.J. (2020). Efectos del coronavirus en el sistema de enseñanza: aumenta la desigualdad de oportunidades educativas en España. Revista de Sociología de la Educación-RASE, 13(2), 114-139. https://doi.org/10.7203/RASE.13.2.17125

Ceballos Marón, N.A. y Sevilla Vallejo, S. (2020). El Efecto del Aislamiento Social por el Covid19 en la Conciencia Emocional y en la Comprensión Lectora. Estudio sobre la Incidencia en Alumnos con Trastornos de Aprendizaje y Menor Acceso a las Nuevas Tecnologías. Revista Internacional de Educación para la Justicia Social, 9(3), 1-13.

CONFAPA. (2020). Crise sanitaria COVID-19. Resultados enquisa respecto do ensino non presencial. CONFAPA. https://cutt.ly/gvOdIMo

Cotino Hueso, L. (2020). La enseñanza digital en serio y el derecho a la educación en tiempos del coronavirus. Revista de educación y derecho, 21(6), 1-29.

https://doi.org/10.1344/REYD2020.21.31283

Díez-Gutiérrez, E.J. (2021, enero, 18). El pleno derecho a la educación solo puede ser presencial. El Diario de la Educación. https://cutt.ly/lvtQeNc

Díez-Gutiérrez, E.J. y Gajardo Espinoza, K. (2020). Educar y evaluar en tiempos de Coronavirus: la situación en España. Multidisciplinary Journal of Educational Research, 10(2), 102-134. http://dx.doi.org/10.17583/remie.2020.5604 
Estadística Junta de Castilla y León (2020, 10 de mayo). Indicadores Sociales CCYL. Junta de Castilla y León. https://estadistica.jcyl.es/web/es/estadisticas-temas/indicadoressociales.html

EU Kids Online. (2018). Los niños y niñas de la brecha digital en España. UNICEF. https://cutt.ly/lvOdHyc

Fundación Telefónica. (2020). Sociedad digital en España 2019. Madrid: Taurus-Fundación Telefónica. https://cutt.ly/cvtvygM

García-García, M.D. (2020). La docencia desde el hogar. Una alternativa necesaria en tiempos del Covid 19. Polo del Conocimiento, 5(4), 304-324.

https://doi.org/10.23857/pc.v5i3.1318

Heppen, J.B., Sorensen, N., Allensworth, E., Walters, K., Rickles, J., Taylor, S.S. y Michelman, V. (2017). The struggle to pass algebra: Online vs. face-to-face credit recovery for at-risk urban students. Journal of Research on Educational Effectiveness, 10(2), 272-296. http://dx.doi.org/10.1080/19345747.2016.1168500

Hernández, J.V. (2020). Impacto de la pandemia SARS-COV2 en nuestros jóvenes. Revista Latinoamericana de Investigación Social, 3(1), 9-14.

Hernández, C.E. y Carpio, N. (2019). Introducción a los tipos de muestreo. ALERTA Revista Científica del Instituto Nacional de Salud, 2(1), 75-79. https://doi.org/10.5377/alerta.v2i1.7535

INE. (2019). Encuesta sobre equipamiento y uso de tecnologías de información y comunicación en los hogares 2019. INE. https://www.ine.es/prensa/tich_2019.pdf

Lacort, J. (2020, abril, 20). La escuela por Internet iba a democratizar la educación, pero el curso forzosamente online está teniendo el efecto opuesto. Xataca. https://cutt.ly/RyrJdGK

Martín Criado, E. y Gómez Bueno, C. (2017). El mito de la dimisión parental. Implicación familiar, desigualdad social y éxito escolar. Cuadernos de Relaciones Laborales, 35(2), 35. https://doi.org/10.5209/CRLA.56777

Martín, J.M. y Rogero, J. (2020, abril, 17). El coronavirus y la asfixia educativa: el confinamiento deja sin protección a la infancia más vulnerable. El diario. https://cutt.ly/iyG9xid

MEFP (2020, 19 de mayo). Estadísticas de la Educación: Datos Básicos. España. Educación y formación profesional. https://www.educacionyfp.gob.es/servicios-al-ciudadano/estadisticas.html

Mendieta Izquierdo, M. (2015). Informantes y muestreo en investigación cualitativa. Investigaciones Andina, 17(30), 1.148-1.150. https://doi.org/10.33132/01248146.65

Ozamiz-Etxebarría, N., Dosil-Santamaría, M., Picaza-Gorrochategui, M. e Idoiaga-Mondragón, N. (2020). Niveles de estrés, ansiedad y depresión en la primera fase del brote del COVID19 en una muestra recogida en el norte de España. Cadernos de Saúde Pública, 36, e00054020. https://doi.org/10.1590/0102-311x00054020

Piña-Ferrer, L. (2020). El COVID 19: Impacto psicológico en los seres humanos. Interdisciplinaria de Ciencias de la Salud. Salud y Vida, 4(7), 188-199. http://doi.org/dzkk

Reips, U.D. (2002). Standards for Internet-based experimenting. Experimental psychology, 49(4), 243-256. http://doi.org/dhf2n6

Samaniego García, E. (2020). Reflexiones sobre la educación telemática en tiempos de coronavirus. Revista AOSMA, 28, 13-19.

Serna, M. (2019). ¿Cómo mejorar el muestreo en estudios de porte medio usando diseños con métodos mixtos? Aportes desde el campo de estudio de elites. Empiria: Revista de metodología de ciencias sociales, 43, 187-210. https://doi.org/10.5944/empiria.43.2019.24305

UNESCO. (2020, 2 de septiembre). UNESCO: COVID-19 Response. UNESCO. https://en.unesco.org/covid19 
UNICEF. (2020, 3 de marzo). El nuevo coronavirus y el derecho a la educación. UNICEF. https://www.unicef.es/educa/blog/nuevo-coronavirus-derecho-educacion

Xu, D. y Jaggars, S.S. (2014). Performance Gaps Between Online and Face-to-Face Courses: Differences Across Types of Students and Academic Subject Areas. The Journal of Higher Education 85(5), 633-659. https://doi.org/10.1080/00221546.2014.11777343

Zubillaga, A. y Gortázar, L. (2020). COVID-19 y educación: problemas, respuestas y escenarios. Madrid: Fundación COTEC. 